\title{
CSResarch Square \\ Linking respiratory disease to microbial makeup of indoor space
}

Xi Fu

Zheyuan Ou

Mei Zhang

Yi Meng

Yanling Li

Jikai Wen

Qiansheng $\mathrm{Hu}$

Xin Zhang

Dan Norbäck

Yiqun Deng

Zhuohui Zhao

Yu Sun

\section{Video Byte}

Keywords: asthma, rhinitis, shotgun metagenomics, high school students, urban/rural, China, Microbiome, respiratory disease, dust, vacuum dust

Posted Date: October 14th, 2021

DOl: https://doi.org/10.21203/rs.3.rs-968203/v1

License: (9) This work is licensed under a Creative Commons Attribution 4.0 International License.

Read Full License 


\section{Abstract}

Since World War II, the prevalence of chronic respiratory diseases such as asthma and rhinitis have increased dramatically around the globe but not at the same rate everywhere. Data suggests that these diseases occur more frequently in urban than in rural areas. To understand why, researchers analyzed the microbial makeup of classroom vacuum dust from nine high schools in China. Their findings revealed significant differences in microbial makeup between urban and rural schools. Urban schools were rich in species from Betaproteobactera, Gammaproteobacteria, and Bacilli, while rural schools were dominated by species from Actinobacteria and Cyanobacteria. Importantly, urban schools were more abundant in potential disease-causing pathogens, whereas rural schools showed DNA evidence of substances that combat inflammatory disease in the human gut. This is the first time researchers have used the tools of microbial metagenomics to compare indoor spaces. While more work is needed to confirm the activity of the microbes detected, the findings offer evidence of the important link between the microbial makeup of indoor spaces and disease. 